\title{
Crystal Structure of a New Heterotrinuclear Complex, $\left[\mathrm{Cu}\left(\mathrm{C}_{2} \mathrm{H}_{8} \mathrm{~N}_{2}\right)_{2}\right]\left[\left\{\mathrm{HB}\left(\mathrm{C}_{3} \mathrm{H}_{3} \mathrm{~N}_{2}\right)_{3}\right\} \mathrm{Fe}(\mathrm{CN})_{3}\right]_{2}$
}

\author{
Shi WANG, Yi-Zhi LI, ${ }^{\dagger}$ Jing-Lin Zuo, ${ }^{\dagger}$ and Xiao-Zeng You \\ Coordination Chemistry Institute, State Key Laboratory of Coordination Chemistry, \\ Nanjing University, Nanjing, 210093, P. R. China
}

\begin{abstract}
The title compound, $\left[\mathrm{Cu}\left(\mathrm{C}_{2} \mathrm{H}_{8} \mathrm{~N}_{2}\right)_{2}\right]\left[\left\{\mathrm{HB}\left(\mathrm{C}_{3} \mathrm{H}_{3} \mathrm{~N}_{2}\right)_{3}\right\} \mathrm{Fe}(\mathrm{CN})_{3}\right]_{2}$, crystallizes in the monoclinic space group $P 2_{1} / c$ with $a=$ 34.924(2) $\AA, b=7.923(2) \AA, c=13.546(2) \AA, \beta=96.201(3)^{\circ}, V=3726.3(11) \AA^{3}, Z=4$. The structure was refined to a final $R$ value of 0.0622 for 4365 reflections $(I>2 \sigma(I))$. The copper atom is in a distorted octahedral environment: two cyano nitrogen atoms in trans positions and four nitrogen atoms from the ethylenediamine molecules. The intramolecular $\mathrm{Fe}(1)-\mathrm{Cu}(1), \mathrm{Fe}(2)-\mathrm{Cu}(1)$ and $\mathrm{Fe}(1)-\mathrm{Fe}(2)$ distances are 5.199, 5.211 and $10.410 \AA$, respectively.
\end{abstract}

(Received February 27, 2004; Accepted May 25, 2004; Published on Web July 30, 2004)

Recently, much interest has been focused on the study of cyanide-containing metal complexes because of their unique magnetic properties. ${ }^{1,2}$ Among these interesting research subjects, low-dimensional as well as polynuclear clusters have attracted special attention. The reason is that such systems can be used to investigate the intermetallic magnetic coupling quantitatively, whereas the lack of an appropriate model for high-dimensional systems creates a problem for quantitative magnetic analysis. In an effort to continue this remarkably

Table 1 Crystal and experimental data

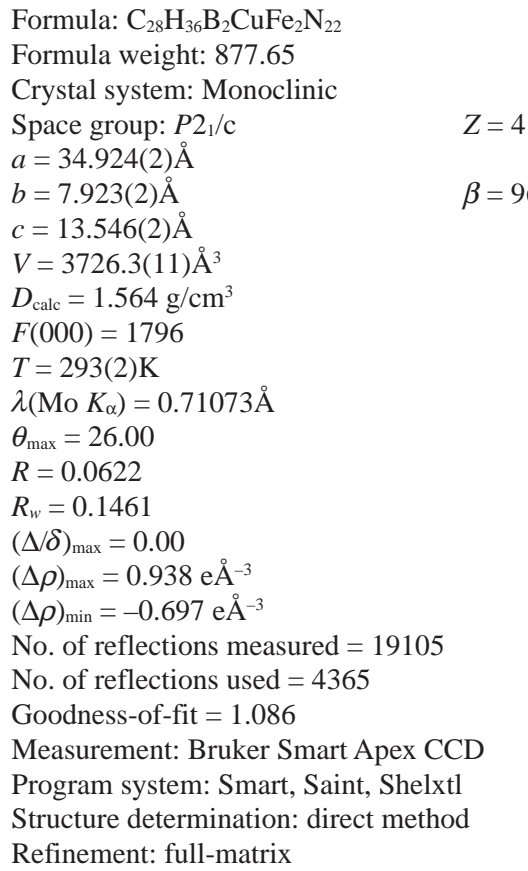

$\doteqdot$ To whom correspondence should be addressed.

E-mail address: zuojl@nju.edu.cn diverse chemistry, we choose the tailored cyanometalate precursor, $\left(\mathrm{Bu}_{4} \mathrm{~N}\right)\left[(\mathrm{Tp}) \mathrm{Fe}(\mathrm{CN})_{3}\right](\mathrm{Tp}=$ tris (pyrazolyl)hydrobo rate; $\mathrm{Bu}_{4} \mathrm{~N}^{+}=$tetrabutylammonium cation), which consists of three $\mathrm{CN}^{-}$groups and the ligand of tris (pyrazolyl)hydroborate (Tp). This compound was also reported by Julve's group as

Table 2 Selected atomic coordinates and equivalent isotropic thermal parameters $U_{\text {eq }}\left(\AA^{2}\right)$ for non-hydrogen atoms

\begin{tabular}{|c|c|c|c|c|}
\hline Atom & $x$ & $y$ & $z$ & $U_{\mathrm{eq}}$ \\
\hline $\mathrm{Cul}$ & $0.2500(1)$ & $0.5006(1)$ & $0.6626(1)$ & $0.037(1)$ \\
\hline $\mathrm{Fe} 1$ & $0.1312(1)$ & $0.2334(1)$ & $0.4555(1)$ & $0.036(1)$ \\
\hline $\mathrm{Fe} 2$ & $0.3687(2)$ & $0.7678(1)$ & $0.8714(1)$ & $0.034(1)$ \\
\hline N1 & $0.0953(1)$ & $0.1429(6)$ & $0.5500(4)$ & $0.043(1)$ \\
\hline $\mathrm{N} 2$ & $0.0568(1)$ & $0.1565(5)$ & $0.5260(3)$ & $0.039(1)$ \\
\hline N3 & $0.0979(1)$ & $0.4355(6)$ & $0.4383(3)$ & $0.036(1)$ \\
\hline $\mathrm{N} 4$ & $0.0601(1)$ & $0.4234(6)$ & $0.4303(3)$ & $0.040(1)$ \\
\hline N5 & $0.0981(1)$ & $0.1345(5)$ & $0.3422(3)$ & $0.037(1)$ \\
\hline N6 & $0.0585(1)$ & $0.1480(5)$ & $0.3432(3)$ & $0.039(1)$ \\
\hline N7 & $0.1819(1)$ & $0.4051(6)$ & $0.6246(4)$ & $0.041(1)$ \\
\hline N8 & $0.1934(1)$ & $0.3470(6)$ & $0.3275(3)$ & $0.043(1)$ \\
\hline N9 & $0.1779(1)$ & $-0.0960(6)$ & $0.4821(4)$ & $0.045(1)$ \\
\hline N10 & $0.4024(1)$ & $0.8669(5)$ & $0.9859(3)$ & $0.035(1)$ \\
\hline N11 & $0.4410(1)$ & $0.8464(5)$ & $0.9891(3)$ & $0.035(1)$ \\
\hline N12 & $0.3999(1)$ & $0.5576(6)$ & $0.8857(3)$ & $0.043(1)$ \\
\hline N13 & $0.4396(1)$ & $0.5747(6)$ & $0.8997(3)$ & $0.043(1)$ \\
\hline N14 & $0.4048(1)$ & $0.8580(6)$ & $0.7817(3)$ & $0.043(1)$ \\
\hline N15 & $0.4441(1)$ & $0.8442(6)$ & $0.8094(4)$ & $0.043(1)$ \\
\hline N16 & $0.3187(1)$ & $0.5882(6)$ & $0.7063(4)$ & $0.044(1)$ \\
\hline N17 & $0.3221(1)$ & $1.0926(6)$ & $0.8479(3)$ & $0.044(1)$ \\
\hline N18 & $0.3052(1)$ & $0.6509(6)$ & $0.9961(3)$ & $0.044(1)$ \\
\hline N19 & $0.2624(1)$ & $0.2616(5)$ & $0.7020(3)$ & $0.043(1)$ \\
\hline $\mathrm{N} 20$ & $0.2631(1)$ & $0.4276(5)$ & $0.5263(3)$ & $0.044(1)$ \\
\hline $\mathrm{N} 21$ & $0.2378(1)$ & $0.7402(5)$ & $0.6230(3)$ & $0.042(1)$ \\
\hline $\mathrm{N} 22$ & $0.2374(1)$ & $0.5702(5)$ & $0.8023(3)$ & $0.039(1)$ \\
\hline $\mathrm{C} 10$ & $0.1627(2)$ & $0.3390(7)$ & $0.5618(4)$ & $0.043(2)$ \\
\hline C11 & $0.1688(2)$ & $0.3069(7)$ & $0.3689(4)$ & $0.043(2)$ \\
\hline $\mathrm{C} 12$ & $0.1608(2)$ & $0.0301(7)$ & $0.4714(4)$ & $0.044(2)$ \\
\hline $\mathrm{C} 22$ & $0.3369(2)$ & $0.6625(7)$ & $0.7639(4)$ & $0.043(2)$ \\
\hline $\mathrm{C} 23$ & $0.3389(2)$ & $0.9684(7)$ & $0.8544(4)$ & $0.043(2)$ \\
\hline C24 & $0.3305(2)$ & $0.6884(7)$ & $0.9516(4)$ & $0.043(2)$ \\
\hline $\mathrm{C} 25$ & $0.2263(2)$ & $0.8386(7)$ & $0.7076(4)$ & $0.045(2)$ \\
\hline $\mathrm{C} 26$ & $0.2172(2)$ & $0.7314(7)$ & $0.7901(4)$ & $0.045(2)$ \\
\hline $\mathrm{C} 27$ & $0.2728(2)$ & $0.1728(7)$ & $0.6215(4)$ & $0.045(2)$ \\
\hline $\mathrm{C} 28$ & $0.2755(2)$ & $0.2614(7)$ & $0.5260(4)$ & $0.045(2)$ \\
\hline B1 & $0.0423(2)$ & $0.2482(8)$ & $0.4253(5)$ & $0.041(2)$ \\
\hline B2 & $0.4567(2)$ & $0.7568(8)$ & $0.9044(5)$ & $0.043(2)$ \\
\hline
\end{tabular}

$U_{\text {eq }}=(1 / 3) \Sigma_{i} \Sigma_{j} U_{i j}\left(a_{i}^{*} a_{j}^{*}\right)\left(\boldsymbol{a}_{i} \cdot \boldsymbol{a}_{j}\right)$ 
Table 3 Selected bond distances $(\AA)$ and angles $\left({ }^{\circ}\right)$

\begin{tabular}{llll}
\hline $\mathrm{Cu}(1)-\mathrm{N}(19)$ & $2.002(4)$ & $\mathrm{Fe}(1)-\mathrm{C}(10)$ & $1.908(6)$ \\
$\mathrm{Cu}(1)-\mathrm{N}(21)$ & $2.005(4)$ & $\mathrm{Fe}(1)-\mathrm{C}(12)$ & $1.914(6)$ \\
$\mathrm{Cu}(1)-\mathrm{N}(20)$ & $2.034(4)$ & $\mathrm{Fe}(1)-\mathrm{C}(11)$ & $1.942(6)$ \\
$\mathrm{Cu}(1)-\mathrm{N}(22)$ & $2.064(4)$ & $\mathrm{Fe}(2)-\mathrm{C}(23)$ & $1.901(6)$ \\
$\mathrm{Cu}(1)-\mathrm{N}(7)$ & $2.494(4)$ & $\mathrm{Fe}(2)-\mathrm{C}(24)$ & $1.915(6)$ \\
$\mathrm{Cu}(1)-\mathrm{N}(16)$ & $2.507(5)$ & $\mathrm{Fe}(2)-\mathrm{C}(22)$ & $1.925(6)$ \\
& & & \\
$\mathrm{C}(10)-\mathrm{N}(7)-\mathrm{Cu}(1)$ & $139.1(4)$ & $\mathrm{N}(19)-\mathrm{Cu}(1)-\mathrm{N}(21)$ & $179.62(19)$ \\
$\mathrm{C}(22)-\mathrm{N}(16)-\mathrm{Cu}(1)$ & $140.1(5)$ & $\mathrm{N}(19)-\mathrm{Cu}(1)-\mathrm{N}(20)$ & $84.79(18)$ \\
$\mathrm{N}(7)-\mathrm{C}(10)-\mathrm{Fe}(1)$ & $178.7(6)$ & $\mathrm{N}(21)-\mathrm{Cu}(1)-\mathrm{N}(20)$ & $95.06(18)$ \\
$\mathrm{N}(8)-\mathrm{C}(11)-\mathrm{Fe}(1)$ & $172.5(5)$ & $\mathrm{N}(19)-\mathrm{Cu}(1)-\mathrm{N}(22)$ & $94.02(18)$ \\
$\mathrm{N}(9)-\mathrm{C}(12)-\mathrm{Fe}(1)$ & $177.9(5)$ & $\mathrm{N}(21)-\mathrm{Cu}(1)-\mathrm{N}(22)$ & $86.13(17)$ \\
$\mathrm{N}(16)-\mathrm{C}(22)-\mathrm{Fe}(2)$ & $173.6(6)$ & $\mathrm{N}(20)-\mathrm{Cu}(1)-\mathrm{N}(22)$ & $178.70(18)$ \\
$\mathrm{N}(17)-\mathrm{C}(23)-\mathrm{Fe}(2)$ & $176.6(5)$ & $\mathrm{N}(19)-\mathrm{Cu}(1)-\mathrm{N}(7)$ & $86.72(17)$ \\
$\mathrm{N}(18)-\mathrm{C}(24)-\mathrm{Fe}(2)$ & $174.0(5)$ & $\mathrm{N}(21)-\mathrm{Cu}(1)-\mathrm{N}(7)$ & $93.63(17)$ \\
$\mathrm{N}(20)-\mathrm{Cu}(1)-\mathrm{N}(7)$ & $91.73(17)$ & $\mathrm{N}(22)-\mathrm{Cu}(1)-\mathrm{N}(7)$ & $88.73(16)$ \\
$\mathrm{N}(19)-\mathrm{Cu}(1)-\mathrm{N}(16)$ & $91.36(17)$ & $\mathrm{N}(21)-\mathrm{Cu}(1)-\mathrm{N}(16)$ & $88.29(17)$ \\
& & &
\end{tabular}

Estimated standard deviations in the least-significant figure are given in parentheses.

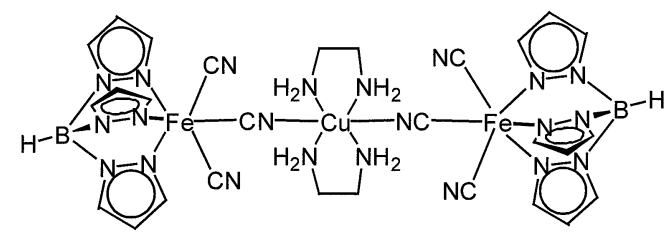

Fig. 1 Chemical diagram.

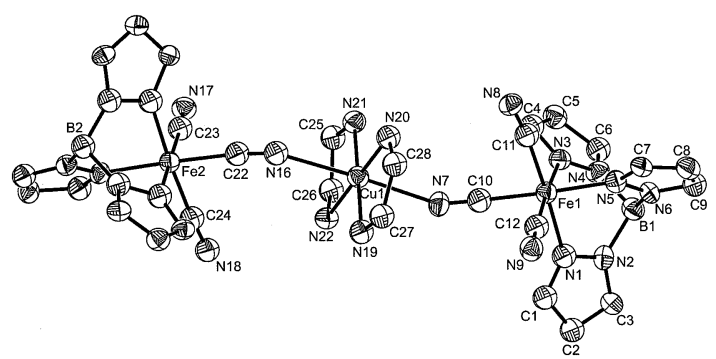

Fig. 2 Molecular structure of the title compound with the atomnumbering scheme. The thermal ellipsoids are drawn at the 50\% probability level.

tetraphenylphosphonium salt recently. ${ }^{3}$

A mixture of ethanol and water was gently layered on the top of a solution of $\left[\mathrm{Cu}(\mathrm{en})_{3}\right] \mathrm{Cl}_{2}$ (en: ethylenediamine) in water. A solution of $\left(\mathrm{Bu}_{4} \mathrm{~N}\right)\left[(\mathrm{Tp}) \mathrm{Fe}(\mathrm{CN})_{3}\right]$ in ethanol was carefully added as a third layer. Crystals were obtained after one week, washed with ethanol, ether and dried in air. An orange crystal obtained in this way was chosen and its structure was determined by single-crystal X-ray diffraction. The crystal and experimental data are given in Table 1 . The residual density maximum is $0.938 \mathrm{e}^{-3}$, which is near $\mathrm{Fe} 1$ atom. All $\mathrm{H}$ atoms were placed in calculated positions and included in the refinement in riding motion approximation with $U_{\text {iso }}=1.2 U_{\text {eq }}$ of the carrier atom. The final fractional atomic coordinates and equivalent isotropic thermal parameter for non-hydrogen atoms are given in Table 2. Selected bond distances and bond angles are listed in Table 3.

The structure consists of neutral trinuclear entities of formula $\left[\mathrm{Cu}(\mathrm{en})_{2}\right]\left[(\mathrm{Tp}) \mathrm{Fe}(\mathrm{CN})_{3}\right]_{2}$ (Fig. 1). In this trinuclear complex, the $\left[(\mathrm{Tp}) \mathrm{Fe}(\mathrm{CN})_{3}\right]^{-}$unit acts as a monodentate ligand through one of its three cyano groups toward a central $\left[\mathrm{Cu}(\mathrm{en})_{2}\right]^{2+}$ motif (Fig. 2). Each iron atom is coordinated by three $\mathrm{Tp}$ nitrogen atoms and three cyano carbon atoms, taking a $\mathrm{C}_{3 \mathrm{v}}$ symmetry. The $\mathrm{Fe}-\mathrm{C}$ (cyano) bond length $(1.901(6)-1.942(6) \AA)$ is in good agreement with those observed in the low-spin $\mathrm{Fe}^{3+}$ unit. ${ }^{3,4}$ The Fe-C-N angles for both terminal $\left(172.5(5)-177.9(5)^{\circ}\right)$ and bridging $\left(173.6(6)-178.7(6)^{\circ}\right)$ cyano groups depart somewhat from strict linearity. The copper atom is octahedrally coordinated through six nitrogen atoms. Four nitrogens from the ethylenediamine molecules form the equatorial plane. The average $\mathrm{Cu}-\mathrm{N}(\mathrm{en})$ distance is $2.026 \AA$. Two cyano nitrogen atoms occupy the axial positions. The average $\mathrm{Cu}-\mathrm{N}$ (cyano) bond distance is $2.501 \AA$, which is much longer than the average $\mathrm{Cu}-\mathrm{N}(\mathrm{en})$ bond length $(2.026 \AA)$. The values of $\mathrm{Cu}(1)-\mathrm{N}(7)-\mathrm{C}(10) \quad\left(139.1(4)^{\circ}\right) \quad$ and $\mathrm{Cu}(1)-\mathrm{N}(16)-\mathrm{C}(22)$ $\left(140.1(5)^{\circ}\right)$ depart clearly from $180^{\circ}$; the angles are similar to the corresponding values found in $[\mathrm{Cu}(\text { dien })]_{3}\left[\mathrm{Fe}(\mathrm{CN})_{6}\right]_{2} \cdot 6 \mathrm{H}_{2} \mathrm{O}$ $($ dien $=$ diethylenetriamine $)\left(138.7\right.$ and $\left.142^{\circ}\right) .{ }^{5}$ Each trinuclear entity is linked to the two adjacent trinuclear entities through hydrogen bonds, with $\mathrm{N}(21)-\mathrm{H}(21 \mathrm{~B}) \cdots \mathrm{N}(9)$ and $\mathrm{N}(19)-\mathrm{H}(19 \mathrm{~A}) \cdots \mathrm{N}(17)$ distances of $2.975(7)$ and 3.025(7) $\mathrm{A}$, respectively.

\section{Acknowledgements}

This work was supported by the Major State Basic Research Development Program (G2000077500) and by the National Natural Science Foundation of China (NSF20201006 and 90101028).

\section{References}

1. K. R. Dunbar and R. A. Heintz, Prog. Inorg. Chem., 1997, 45, 283.

2. H. Ōkawa and M. Ohba, Bull. Chem. Soc. Jpn., 2002, 75, 1191.

3. R. Lescouëzec, J. Vaissermann, F. Lloret, M. Julve, and M. Verdaguer, Inorg. Chem., 2002, 41, 5943.

4. H. Oshio, O. Tamada, H. Onodera, T. Ito, T. Ikoma, and S. Tero-Kubota, Inorg. Chem., 1999, 38, 5686.

5. H.-Z. Kou, D.-Z. Liao, P. Cheng, Z.-H. Jiang, S.-P. Yan, G.-L. Wang, X.-K. Yao, and H.-G. Wang, J. Chem. Soc., Dalton Trans., 1997, 1503. 